\title{
Mechanochemical synthesis of aromatic infrared band carriers
}

\section{The top-down chemistry of interstellar carbonaceous dust grain analogues}

\author{
E. Dartois ${ }^{1}$, E. Charon ${ }^{2}$, C. Engrand ${ }^{3}$, T. Pino ${ }^{1}$, and C. Sandt ${ }^{4}$ \\ ${ }^{1}$ Institut des Sciences Moléculaires d'Orsay, CNRS, Université Paris-Saclay, Bât 520, Rue André Rivière, 91405 Orsay, France \\ e-mail: emmanuel.dartois@universite-paris-saclay.fr \\ ${ }^{2}$ Nanosciences et Innovation pour les Matériaux, la Biomédecine et l’Énergie, CEA, CNRS, Université Paris-Saclay, CEA Saclay, \\ 91191 Gif-sur-Yvette, France \\ ${ }^{3}$ Laboratoire de Physique des 2 Infinis Irène Joliot-Curie, CNRS/IN2P3, Université Paris-Saclay, 91405 Orsay, France \\ ${ }^{4}$ Synchrotron Soleil, L'Orme des Merisiers, BP 48 Saint Aubin, 91192 Gif-sur-Yvette Cedex, France
}

Received 13 February 2020 / Accepted 24 March 2020

\begin{abstract}
Context. Interstellar space hosts nanometre- to micron-sized dust grains, which are responsible for the reddening of stars in the visible. The carbonaceous-rich component of these grain populations emits in infrared bands that have been observed remotely for decades with telescopes and satellites. They are a key ingredient of Galactic radiative transfer models and astrochemical dust evolution. However, except for $\mathrm{C}_{60}$ and its cation, the precise carriers for most of these bands are still unknown and not well reproduced in the laboratory. Aims. In this work, we aim to show the high-energy mechanochemical synthesis of disordered aromatic and aliphatic analogues provides interstellar relevant dust particles.

Methods. The mechanochemical milling of carbon-based solids under a hydrogen atmosphere produces particles with a pertinent spectroscopic match to astrophysical observations of aromatic infrared band (AIB) emission, linked to the so-called astrophysical polycyclic aromatic hydrocarbon hypothesis. The $\mathrm{H} / \mathrm{C}$ ratio for the analogues that best reproduce these astronomical infrared observations lies in the $5 \pm 2 \%$ range, potentially setting a constraint on astrophysical models. This value happens to be much lower than diffuse interstellar hydrogenated amorphous carbons, another Galactic dust grain component observed in absorption, and it most probably provides a constraint on the hydrogenation degree of the most aromatic carbonaceous dust grain carriers. A broad band, observed in AIBs, evolving in the $1350-1200 \mathrm{~cm}^{-1}(7.4-8.3 \mu \mathrm{m})$ range is correlated to the hydrogen content, and thus the structural evolution in the analogues produced.

Results. Our results demonstrate that the mechanochemical process, which does not take place in space, can be seen as an experimental reactor to stimulate very local energetic chemical reactions. It introduces bond disorder and hydrogen chemical attachment on the produced defects, with a net effect similar to the interstellar space very localised chemical reactions with solids. From the vantage point of astrophysics, these laboratory interstellar dust analogues will be used to predict dust grain evolution under simulated interstellar conditions, including harsh radiative environments. Such interstellar analogues offer an opportunity to derive a global view on the cycling of matter in other star forming systems.
\end{abstract}

Key words. astrochemistry - dust, extinction - ISM: lines and bands - planetary nebulae: general - infrared: ISM

\section{Introduction}

Our Galaxy hosts carbonaceous dust grains displaying emission and absorption bands observed by telescopes in the infrared.

The emission in the diffuse interstellar medium of these bands, dominated by an aromatic vibrational character, also called AIBs (aromatic infrared bands), has led to the so-called polycyclic aromatic hydrocarbon (PAH) hypothesis. Under this theory, the observed emission is related to the infrared fluorescence emission mechanism of PAH-like molecules (Leger \& Puget 1984; Allamandola et al. 1985), following energetic photon absorption, although no unique PAH has been identified in the mid-infrared so far. Apart from the recent attribution of a few specific infrared emission bands to the $C_{60}$ and possibly $C_{70}$ fullerene molecules in some sources (Sellgren et al. 2009, 2010; Cami et al. 2010), the carriers of the AIBs remain elusive. The emission bands have been categorised in different classes ( $\mathcal{A}$ to $\mathcal{D})$ following the ascertainment of band profiles and center position variations, ensuing from a phenomenological deconvolution of the observations. (Peeters et al. 2002; van Diedenhoven et al. 2004; Matsuura et al. 2007; Sloan et al. 2007; Keller et al. 2008; Boersma et al. 2008; Pino et al. 2008; Acke et al. 2010; Carpentier et al. 2012; Gadallah et al. 2013). In the late classes of infrared emission spectra (so-called $C$ and $\mathcal{D}$ ), a mix between an aromatic and aliphatic character is observed. Class $\mathcal{A}$ sources are dominated by aromatic bands, whereas classes $C$ and $\mathcal{D}$ harbour a pronounced aliphatic character. Most of the interstellar hydrocarbons are injected through the late phases of stellar evolution, post asymptotic giant branch (AGB) and protoplanetary nebula (PPN), which often display the class $C$ and $\mathcal{D}$ spectral character, and are eventually processed later in the ISM. In absorption, bands at $3.4,6.85$, and $7.25 \mu \mathrm{m}$ are also observed in the diffuse interstellar medium (ISM) of our Galaxy, as well as in extragalactic ISM, and can be well represented by a material with a significant amount of aliphatic character, also called HAC or a-C:H, which is a family of hydrogenated amorphous carbons (e.g. Allen \& Wickramasinghe 1981; Duley \& Williams 1983; Mason et al. 2004; Risaliti et al. 2006; Dartois et al. 2007; 
Table 1. Observation summary.

\begin{tabular}{|c|c|c|c|c|c|}
\hline Object & Right Ascension & Declination & Obs. identifier or reference & Class & Type (Simbad) \\
\hline SMC 2MJ004441 & 00h 44m 41.05s & $-73 \mathrm{~d} 21^{\prime} 36.4^{\prime \prime}$ & Spitzer AORKEY 27525120 & $\mathcal{D}$ & Protoplanetary nebula \\
\hline IRAS 04296+3429 & $04 \mathrm{~h} 32 \mathrm{~m} \mathrm{56.976s}$ & $+34 \mathrm{~d} 36^{\prime} 12.51^{\prime \prime}$ & $\begin{array}{l}\text { Spitzer AORKEY } 4116736 \\
\text { UKIRT; Geballe et al. (1992) }\end{array}$ & $\mathcal{C}, \mathcal{D}$ & Protoplanetary nebula \\
\hline IRAS 05073-6752 & 05h $07 \mathrm{~m} 13.920 \mathrm{~s}$ & $-67 \mathrm{~d} 48^{\prime} 46.73^{\prime \prime}$ & Spitzer AORKEY 24317184 & $\mathcal{D}$ & Protoplanetary nebula \\
\hline IRAS 05110-6616 & $05 \mathrm{~h} 11 \mathrm{~m} 10.639 \mathrm{~s}$ & $-66 \mathrm{~d} 12^{\prime} 53.63^{\prime \prime}$ & Spitzer AORKEY 25992704 & $\mathcal{D}$ & Post AGB \\
\hline IRAS S05211-6926 & $05 \mathrm{~h} 20 \mathrm{~m} \mathrm{43.58s}$ & $-69 \mathrm{~d} 23^{\prime} 41.4^{\prime \prime}$ & Spitzer AORKEY 27985920 & $\mathcal{D}$ & Pulsating star \\
\hline IRAS Z05259-7052 & $05 \mathrm{~h} 25 \mathrm{~m} 20.75 \mathrm{~s}$ & $-70 \mathrm{~d} 50^{\prime} 07.3^{\prime \prime}$ & Spitzer AORKEY 25996032 & $\mathcal{D}$ & Variable star \\
\hline IRAS 05289-6617 & 05h $29 \mathrm{~m} 02.412 \mathrm{~s}$ & $-66 \mathrm{~d} 15^{\prime} 27.77^{\prime \prime}$ & Spitzer AORKEY 11239680 & $\mathcal{D}$ & Carbon star \\
\hline IRAS $05341+0852$ & $05 \mathrm{~h} 36 \mathrm{~m} 55.050 \mathrm{~s}$ & $+08 \mathrm{~d} 54^{\prime} 08.65^{\prime \prime}$ & $\begin{array}{l}\text { Spitzer AORKEY } 4896512 \\
\text { UKIRT; Joblin et al. (1996) }\end{array}$ & $\mathcal{D}$ & Protoplanetary nebula \\
\hline IRAS 06111-7023 & 06h $10 \mathrm{~m} \mathrm{32.008s}$ & $-70 \mathrm{~d} 24^{\prime} 40.72^{\prime \prime}$ & Spitzer AORKEY 19013120 & $\mathcal{D}$ & Star \\
\hline IRAS 13416-6243 & $13 \mathrm{~h} 45 \mathrm{~m} 07.65 \mathrm{~s}$ & $-62 \mathrm{~d} 58^{\prime} 19.0^{\prime \prime}$ & ISO TDT 62803904 & $C$ & Protoplanetary nebula \\
\hline IRAS 15038-5533 & $15 \mathrm{~h} 07 \mathrm{~m} 34.79 \mathrm{~s}$ & $-55 \mathrm{~d} 44^{\prime} 50.8^{\prime \prime}$ & Spitzer AORKEY 17936384 & $\mathcal{D}$ & Far-IR source \\
\hline IRAS $20000+3239$ & $20 \mathrm{~h} 01 \mathrm{~m} 59.513 \mathrm{~s}$ & $+32 \mathrm{~d} 47^{\prime} 32.81^{\prime \prime}$ & $\begin{array}{l}\text { ISO TDT } 18500531 \\
\text { Spitzer AORKEY } 4897792\end{array}$ & $\mathcal{D}$ & Variable star \\
\hline AFGL 2688 & $21 \mathrm{~h} 02 \mathrm{~m} 18.79 \mathrm{~s}$ & $+36 \mathrm{~d} 41^{\prime} 37.4^{\prime \prime}$ & $\begin{array}{l}\text { ISO TDT } 35102563 \\
\text { UKIRT; Geballe et al. (1992) }\end{array}$ & $C$ & Protoplanetary nebula \\
\hline IRAS $22272+5435$ & $22 \mathrm{~h} 29 \mathrm{~m} 10.375 \mathrm{~s}$ & $+54 \mathrm{~d} 51^{\prime} 06.34^{\prime \prime}$ & $\begin{array}{l}\text { ISO TDT } 26302115 \\
\text { Subaru; Goto et al. (2003) }\end{array}$ & $\mathcal{D}$ & Protoplanetary nebula \\
\hline IRAS 23304+6147 & $23 \mathrm{~h} 32 \mathrm{~m} 44.785 \mathrm{~s}$ & $+62 \mathrm{~d} 03^{\prime} 49.08^{\prime \prime}$ & $\begin{array}{l}\text { ISO TDT } 39601867 \\
\text { Spitzer AORKEY } 14401024\end{array}$ & $\mathcal{D}$ & Protoplanetary nebula \\
\hline
\end{tabular}

Dartois \& Muñoz-Caro 2007; Imanishi et al. 2010, and references therein). However, the AIB emission spectra are not a simple linear combination of aliphatic (such as the a-C:H observed in absorption in the diffuse ISM) and aromatic (class $\mathcal{A}$ observed in emission) spectra. They show a spectral evolution of vibrational modes, including the shift of the $\mathrm{C}=\mathrm{C}$ mode from 6.2 to $6.3 \mu \mathrm{m}$ from class $\mathcal{A}$ to $C$ (e.g. van Diedenhoven et al. 2004), and broadening and shifting back to lower wavelengths in class $\mathcal{D}$. Unlike classes $\mathcal{A}-\mathcal{B}$ with two bands in the 7.6 to $8.2 \mu \mathrm{m}$ range, classes $C-\mathcal{D}$ show a broad band (Szczerba et al. 2005; Matsuura et al. 2014), with the class $\mathcal{D}$ one peaking at a shorter wavelength. The out of plane, predominantly aromatic, $\mathrm{CH}$ vibration patterns (from 11 to $13 \mu \mathrm{m}$ ) are generally difficult to reproduce with dust analogues in the laboratory. In addition to chemically pure PAH studies, a large range of dust grain analogues have been tailored using lasers, flames, VUV continuum sources, and/or plasmas in the laboratory to tackle this identification issue (Carpentier et al. 2012; Schnaiter et al. 1999; Jäger et al. 2006; Mennella et al. 1999; Dartois et al. 2005; Furton et al. 1999; Biennier et al. 2009). Among the issues faced by laboratory synthesis of interstellar dust analogues is the ability to produce an environment that is not homogeneous for the entire batch of analogues produced (i.e. localised modifications), as many processes in the interstellar medium will only affect grain (surfaces) moieties considering, for example, hydrogen or radical accretion/addition, cosmic ray impact, grain-grain shocks, etc. As a consequence, a large inhomogeneity at the nanometre scale between adjacent constitutive elements making the grain may be expected, introducing many local defects that will influence the nature and spectroscopic properties of such small dust grains. In this study, we address a new non-homogeneous, non-bottom-up shock approach. We used a mechanochemical synthesis method under a pressurised hydrogen atmosphere, to produce laboratory interstellar dust grain analogues to explain the infrared emission spectra of the remotely observed aliphatic and aromatic mixed interstellar dust grains observed through infrared emission bands.

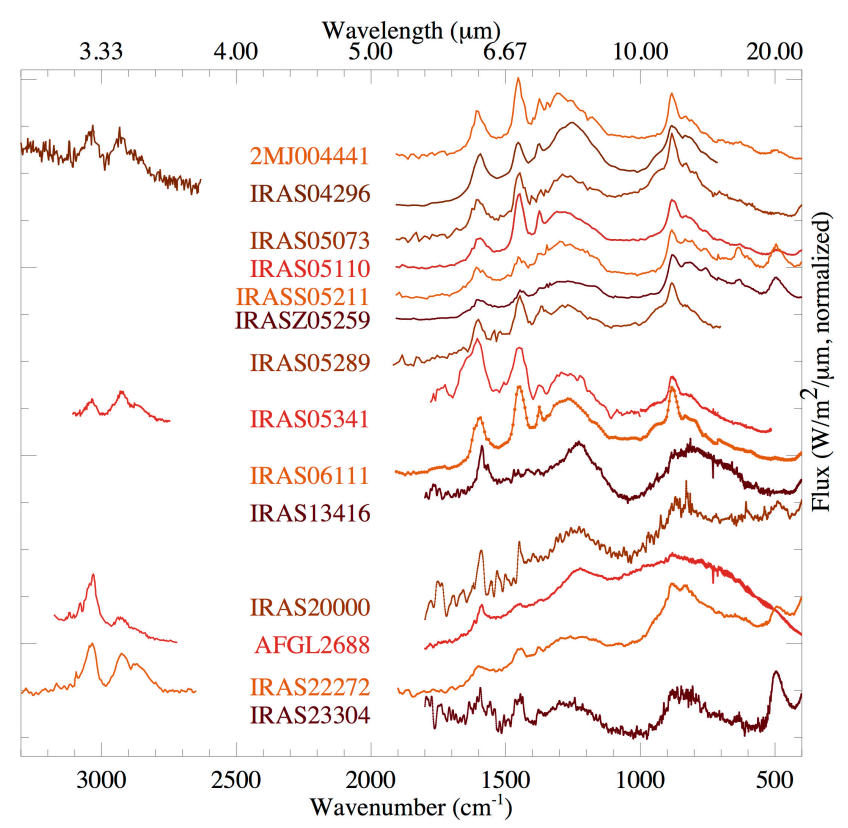

Fig. 1. Astronomical observations of class $C$ and $\mathcal{D}$ sources, displaying characteristic infrared emission bands.

\section{Observations}

Astronomical spectra were retrieved from the ISO $^{1}$ and Spitzer ${ }^{2}$ satellite databases, after a selection from the published literature on clear detections of $C$ and $\mathcal{D}$ classes (Matsuura et al. 2014; Sloan et al. 2007; Szczerba et al. 2005). The existing groundbased $L$-band spectra (in the $2600-3300 \mathrm{~cm}^{-1}$ range) were

\footnotetext{
1 https://www. cosmos.esa. int/web/iso/access-thearchive

2 http://archive.spitzer.caltech.edu/ and https://cassis.sirtf.com/
} 

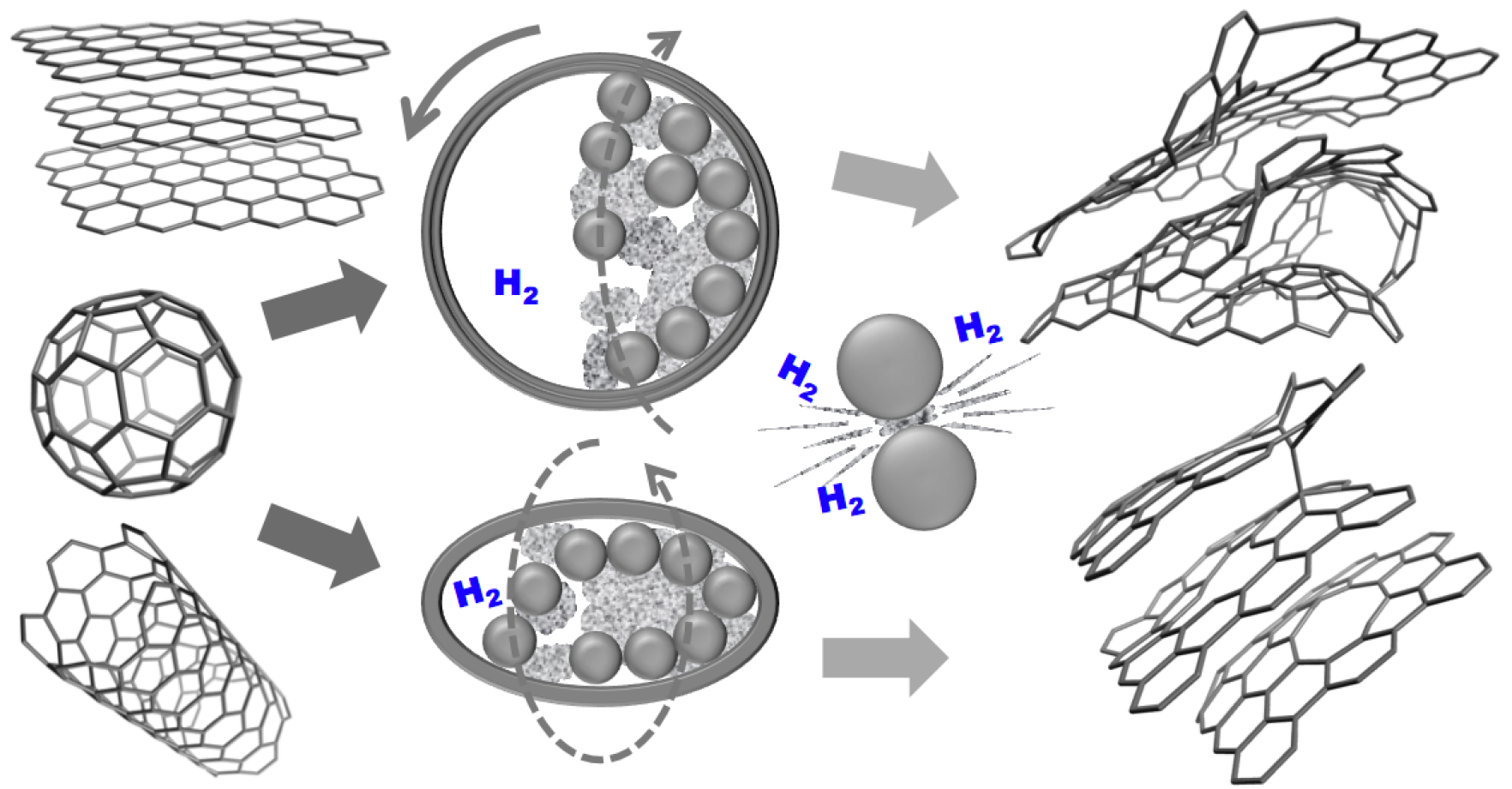

Fig. 2. Schematic representation of the top down mechanochemical process performed with a planetary ball mill (upper) and high-energy mill (lower). The milling impacts and shattering modify the size or crosslink graphite grains, flakes, carbon nanotubes and fullerenes down to the nanometre scale, and a defect-induced chemistry takes place in the pressurised hydrogen-reducing atmosphere.

taken from the literature for IRAS 04296-3429 and AFGL2688 (Geballe et al. 1992), IRAS 05341+0852 (Joblin et al. 1996), and IRAS 22272+5435 (Goto et al. 2003) to complement the spectra at lower wavelengths. A summary of the targets is given in Table 1. The observed spectra are shown in Fig. 1, each one normalised by a scaling factor for clarity.

\section{Experiments}

Several experiments were conducted to produce analogues by mechanochemical milling and analyse them by spectroscopic means, electron microscopy, and elemental analysis.

\subsection{Mechanochemical milling}

Different (hydrogen-free) carbonaceous solid precursors (99.995\% pure graphite or mesoporous activated carbon; Sigma Aldrich; NanoGraphite, Graphene Supermarket; fullerene $\mathrm{C}_{60}$ 99.9+\%, purified, SES research; Multi-wall carbon nanotubes (MWCNT) $98 \%$ pure $<8 \mathrm{~nm}$ outer diameter, carbon nanotubes plus; carbon black nanopowder, average diameter $13 \mathrm{~nm}$, PlasmaChem) were inserted in vacuum-tight bowls. The choice of different carbon precursors was made to test to what extent the initial structure influences the results. The bowls were adapted by in-house connections to firstly be evacuated to a secondary vacuum $\left(<10^{-4}\right.$ mbar $)$ and then pressurised with hydrogen gas before being placed in a Retsch PM100 planetary ( $250 \mathrm{ml}$ or $500 \mathrm{ml}$ bowls) or a Retsch high-energy Emax $(50 \mathrm{ml}$ bowls) ball mill. In the planetary ball mill, the bowl containing grinding balls made of the same material as the bowl is locked at an eccentric position on a rotating sun wheel. The jar and the wheel rotate in opposite directions. The accelerated balls' impacts and frictions at high relative speeds transfer high energy to the solid particles within the bowl. In the Emax ball mill, the grinding jar's oval geometry and higher rotating speeds transfer even higher energy to the particles. A sketch showing the milling process, bowl shapes, and movements is given in Fig. 2. During milling, the bowl temperature never exceeded $80^{\circ} \mathrm{C}$ for the PM100 experiments and was controlled and kept below $50{ }^{\circ} \mathrm{C}$ for the Emax one, ensuring the modifications could not be induced by a high thermostatic temperature. After milling, the samples were extracted in an inflatable polyethylene glove box, evacuated and refilled with Ar or $\mathrm{N}_{2}$, in order to minimise oxygen contamination. The samples were transferred and curated in sealed glass vials placed in an evacuated desiccator. One of the main contaminations of a long-term milling process comes from the abrasion of the bowl itself, producing (nano-) particles that mix within the agglomerated carbonaceous dust particles. The strategy, in order to properly evaluate the impact of the bowl media on the resultant dust particles produced, was to conduct experiments using, when available, different bowl materials and stiffnesses (stainless steel, hardened steel, tungsten carbide, and zirconium oxide). The infrared spectra and electron images on the final products were compared. The balls-to-powder-ratio (BPR), meaning the ratio of the milling balls to sample mass, determining the number and energy of the impacts, varied between 14 and 174. The higher this ratio, the more processed the sample should be. However, it also significantly increases ball-to-ball impacts and shattering, and thus the level of milling medium contamination. A too low BPR does not process the sample efficiently. BPRs centred around 50 seemed a good compromise experimentally. A summary of the performed experiments is given in Table 2.

\subsection{Infrared and Raman measurements}

Infrared measurements were performed by flattening the samples between two diamond windows. Some of the samples were recorded with an In10 Bruker infrared microscope located on the SMIS synchrotron SOLEIL beam line. The infrared spot size was optimised to cover homogeneous flat parts of 
Table 2. Experiment summary.

\begin{tabular}{|c|c|c|c|c|c|c|c|}
\hline Exp. & Precursor (weight) & $\operatorname{BPR}^{(a)}$ & $\begin{array}{l}\mathrm{H}_{2}{ }^{(b)} \\
\text { (bar) }\end{array}$ & $\operatorname{RPM}^{(c)}$ & Time $^{(d)}$ & $\begin{array}{l}\mathrm{H} / \mathrm{C} \\
\text { (at. \%) }\end{array}$ & $\operatorname{HRTEM}^{(e)}$ \\
\hline \multicolumn{8}{|c|}{ Stainless steel } \\
\hline sst-a & Graphite $(1.3 \mathrm{~g})$ & 94 & 3.5 & 400 & $90 \mathrm{~h}$ & 4.27 & - \\
\hline sst-b & Graphite (1.7 g) & 72 & 4.3 & 400 & $43 \mathrm{~h} 30$ & 3.52 & - \\
\hline sst-c & Mesoporous C (5 g) & 25 & 13 & 400 & $44 \mathrm{~h} 30$ & - & - \\
\hline sst-d & Mesoporous C (5 g) & 25 & 16 & 400 & $45 \mathrm{~h} 45$ & - & - \\
\hline sst-e ${ }^{(f)}$ & Mesoporous C (5 g) & 25 & 16 & 400 & $45 \mathrm{~h} 45$ & - & - \\
\hline sst-f & Graphite $(2.5 \mathrm{~g})$ & 49 & 20 & 400 & $71 \mathrm{~h} 30$ & 6.38 & $\mathrm{y}$ \\
\hline sst-g & Fullerene $(2.5 \mathrm{~g})$ & 49 & 20 & 400 & $100 \mathrm{~h}$ & 5.34 & $\mathrm{y}$ \\
\hline sst-h & MWCNT (2.5 g) & 46 & 25 & 400 & $155 \mathrm{~h}$ & - & $\mathrm{y}$ \\
\hline sst-i1 & Graphite (1.33 g) & 50 & 20 & 1500 & Oh30 & 1.12 & - \\
\hline sst-i2 & Graphite (1.33 g) & 50 & 20 & 1500 & $2 \mathrm{~h}$ & 2.85 & - \\
\hline sst-i3 & Graphite (1.33 g) & 50 & 20 & 1500 & $8 \mathrm{~h}$ & 3.63 & - \\
\hline sst-j1 & Graphite (1.57 g) & 53 & 20 & 1500 & Oh30 & 2.48 & - \\
\hline sst-j2 & Graphite (1.57 g) & 53 & 20 & 1500 & $2 \mathrm{~h}$ & 4.59 & - \\
\hline sst-j3 & Graphite (1.57g) & 53 & 20 & 1500 & $8 \mathrm{~h}$ & 4.41 & - \\
\hline sst-k & Fullerene (1.33 g) & 50 & 20 & 1500 & $9 \mathrm{~h}$ & 3.00 & - \\
\hline sst-11 & MWCNT (1.31 g) & 51 & 20 & 1500 & $1 \mathrm{~h}$ & 1.82 & - \\
\hline sst-12 & MWCNT (1.31 g) & 51 & 20 & 1500 & $3 \mathrm{~h}$ & 3.19 & - \\
\hline sst-13 & MWCNT (1.31 g) & 51 & 20 & 1500 & $9 \mathrm{~h}$ & 3.41 & - \\
\hline sst-m & Carbon Black (1.32 g) & 51 & 20 & 1500 & $9 \mathrm{~h}$ & - & - \\
\hline \multicolumn{8}{|c|}{ Hardened steel } \\
\hline hst-a1 & Graphite (7.5 g) & 83 & 23 & 400 & $5 \mathrm{~h}$ & 2.83 & $\mathrm{y}$ \\
\hline hst-a2 & Graphite $(7.5 \mathrm{~g})$ & 83 & 23 & 400 & $17 \mathrm{~h}$ & 5.13 & $\mathrm{y}$ \\
\hline hst-a3 & Graphite (7.5 g) & 83 & 23 & 400 & $48 \mathrm{~h}$ & 4.42 & $\mathrm{y}$ \\
\hline hst-b & Graphite (1 g) & 132 & 20 & 400 & $8 \mathrm{~h}$ & - & - \\
\hline hst-c & Graphite (18g) & 37 & 20 & 400 & $72 \mathrm{~h}$ & - & - \\
\hline hst-d & NanoGraphite (15 g) & 14 & 25 & 400 & $234 \mathrm{~h}$ & - & - \\
\hline hst-e & Fullerene $(5 \mathrm{~g})$ & 64 & 25 & 400 & $100 \mathrm{~h}$ & 4.00 & $\mathrm{y}$ \\
\hline hst-f & Carbon black (5 g) & 81 & 17 & 400 & $96 \mathrm{~h}$ & - & $\mathrm{y}$ \\
\hline \multicolumn{8}{|c|}{ Tungsten carbide } \\
\hline cw-a1 & Graphite (5g) & 31 & 20 & 400 & $8 \mathrm{~h}$ & 1.69 & $\mathrm{y}$ \\
\hline $\mathrm{cw}-\mathrm{a} 2$ & Graphite (5g) & 31 & 20 & 400 & $20 \mathrm{~h}$ & 2.38 & $\mathrm{y}$ \\
\hline cw-a3 & Graphite (5 g) & 31 & 20 & 400 & $100 \mathrm{~h}$ & 3.93 & $\mathrm{y}$ \\
\hline cw-b & MWCNT $(7.5 \mathrm{~g})$ & 70 & 25 & 400 & $78 \mathrm{~h}$ & - & - \\
\hline $\mathrm{cW}-\mathrm{c}$ & Fullerene $(3 \mathrm{~g})$ & 174 & 20 & 400 & $26 \mathrm{~h}$ & - & $\mathrm{y}$ \\
\hline \multicolumn{8}{|c|}{ Zirconium oxide } \\
\hline $\mathrm{zr}-\mathrm{a} 1$ & Graphite $(2.5 \mathrm{~g})$ & 31 & 23 & 400 & $4 \mathrm{~h}$ & - & - \\
\hline $\mathrm{zr}-\mathrm{a} 2$ & Graphite $(2.5 \mathrm{~g})$ & 31 & 23 & 400 & $24 \mathrm{~h}$ & - & - \\
\hline zr-a3 & Graphite $(2.5 \mathrm{~g})$ & 31 & 23 & 400 & $131 \mathrm{~h}$ & 3.55 & $\mathrm{y}$ \\
\hline $\mathrm{zr}-\mathrm{b}$ & Fullerene (1.1 g) & 70 & 23 & 500 & $91 \mathrm{~h}$ & 3.00 & $\mathrm{y}$ \\
\hline
\end{tabular}

Notes. ${ }^{(a)}$ Ball-to-powder ratio, i.e. weight of the milling balls with respect to the milled sample; ${ }^{(b)}$ input pressure before milling; ${ }^{(c)}$ revolutions per minute; ${ }^{(d)}$ milling time; ${ }^{(e)}$ High-Resolution Transmission Electron Microscope; ${ }^{(f)} \mathrm{HCl}$ washed after milling.

the samples (typically 10 to $200 \mu \mathrm{m}$ projected aperture). The spectra were recorded with a sensitive cooled (MCT) detector covering the $4000-650 \mathrm{~cm}^{-1}$ range, and, when possible, a room temperature detector (DTGS) to extend the (less sensitive) measurements down to $400 \mathrm{~cm}^{-1}$. Other samples were measured using a Nicolet Continuum XL IR microscope located at the Laboratoire de Physique des deux Infinis Irène JoliotCurie and recorded with a sensitive cooled (MCT) detector covering the $4000-700 \mathrm{~cm}^{-1}$ range and when possible another less sensitive extended (MCT) detector to cover down to about $500 \mathrm{~cm}^{-1}$.

A Thermo Fisher DXR spectrometer located on the SMIS synchrotron SOLEIL beam line was used to record a Raman spectrum for the samples measured in the infrared, using a laser source at $532 \mathrm{~nm}$ at minimum power (100 $\mu \mathrm{W}$ on sample) to prevent any sample alteration. About ten scans with integration times of $20 \mathrm{~s}$ each were co-added for each spectrum. The spot size of the Raman spectra is of the order of a micron. 


\subsection{High-resolution transmission electron microscopy}

Milled sample extracts were diluted in pure ethanol solvent, and a drop was transferred to lacey carbon films supported by $3 \mathrm{~mm}$ TEM grids. Once the solvent evaporated, the grids were mounted in a JEOL JEM-2011 HRTEM microscope operating at an acceleration voltage of $200 \mathrm{kV}$, from the technical platform managed by the Institut des Matériaux de Paris Centre (IMPC) from the CNRS and Sorbonne Université. The microscope is equipped with a GATAN system ORIUS SC100 $(4008 \times 2672$ pixels $)$ CCD camera. Low magnifications $(10000-50000 \times)$ were used for imaging the grain morphologies, and the inclusion of contamination by milling ball material chips that were incorporated in the samples by the milling process. Higher magnification (100000$800000 \times$ ) were used to visualise the profiles of the atomic planes parallel to the incidental beam, and thus the evolution of the nanostructure. To minimise any overlap of the fringes, we selected the thinnest edges in the images at high magnification.

\subsection{Elemental analysis}

The hydrogen and carbon contents of a subset of the produced samples were determined by the SGS company and are reported in Table 2. They were measured after complete combustion in oxygen at $1150{ }^{\circ} \mathrm{C}$ in a helium flux of several $\mathrm{mg}$ of material, by sequentially analysing the resulting combustion gases by thermal conductivity.

\section{Results}

The infrared spectra of a sample set of the produced interstellar analogues by planetary ball milling graphite under a pressurised hydrogen atmosphere with various milling media (stainless steel, hardened steel, tungsten carbide, zirconium oxide) are shown in Fig. 3. Samples were extracted at different milling times to follow the evolution of the transformation. The spectra were continuum subtracted. Electron microscope images of minute amounts of the extracted samples at different milling times, for the hardened steel experiment, are shown in Fig. 4 and were recorded at various spatial scales down to the nanometre range. The images show large agglomerates of particles modified at the nanometre scale. Some coated steel nanoparticles are observed, embedded within the dominating carbon phase, ripped from the bowl and/or steel balls during the violent milling. The images show that the ordered graphite structure is affected even after the first milling phases, and any turbostratic structure totally disappeared at long milling times. Even in the absence of a hydrogen atmosphere, the mechanical milling is known to generate disordered carbon (e.g. Salver-Disma et al. 1999). The addition of hydrogen to the mechanically produced carbon defects induces the saturation of reactive sites (e.g. Francke et al. 2005).

Raman spectra of a sample set of the produced interstellar analogues were recorded and are shown in Fig. 5. The pristine graphite used for the controlled atmosphere milling is also shown, as well as a carbon nanopowder spectrum, literature spectra of multi-layer graphene (Ilyin et al. 2011) and graphene nanoribbons (Jovanović et al. 1996) for comparison. Different bands are observed. The $G$ band $\left(\sim 1580-1600 \mathrm{~cm}^{-1}\right)$, which is the so-called "graphite" band, is in fact present in all $\mathrm{sp}^{2}$ carbons. The $D$ band $\left(\sim 1350 \mathrm{~cm}^{-1}\right)$ is the Raman signature of disorder in $\mathrm{sp}^{2}$ systems. The $D^{\prime}$ band $\left(\sim 1620 \mathrm{~cm}^{-1}\right)$ is best observed in the zr-a1 spectrum where the $G$ band displays an asymmetry that is clearly the sum of two bands. A $D_{3}$ band $\left(\sim 1500 \mathrm{~cm}^{-1}\right)$ fills the region between the $D$ and $G$ bands,

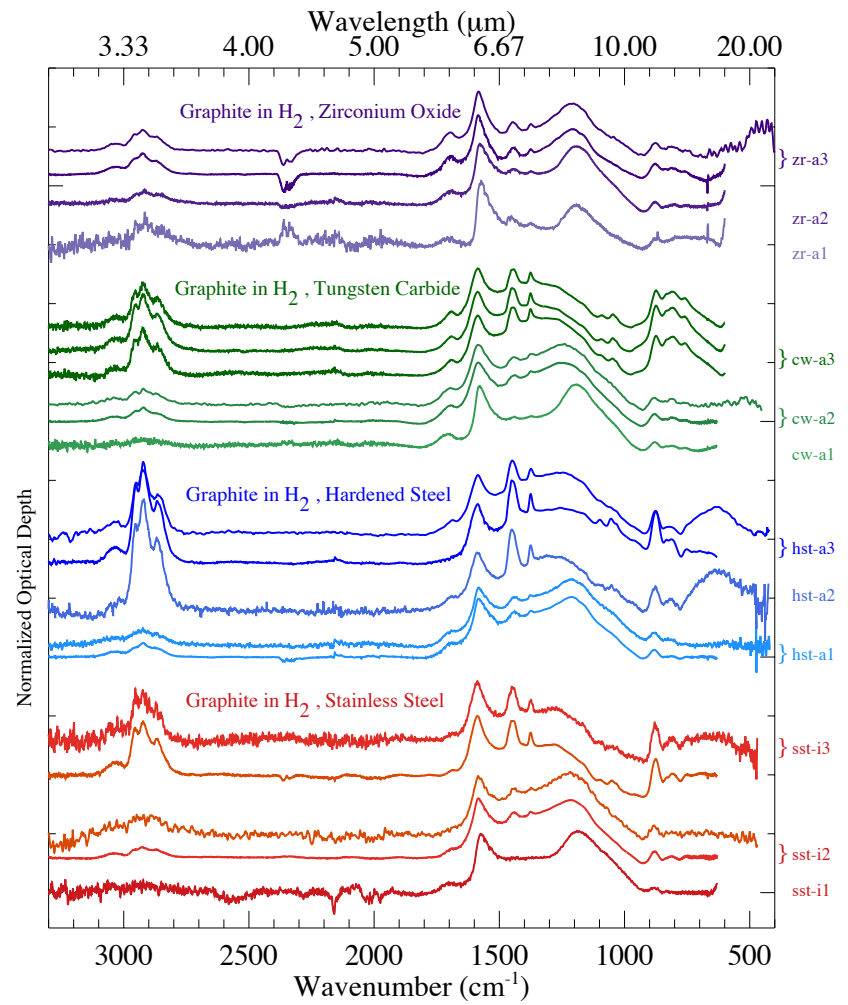

Fig. 3. Infrared spectra of a sample set of planetary ball-milled graphite under a pressurised hydrogen atmosphere in function of milling time. The millings were performed in bowls made of stainless steel, hardened steel, tungsten carbide, and zirconium oxide. The spectra were continuum subtracted.

attributed to the development of an amorphous carbon phase (e.g. Tallant et al. 1998; Sadezky et al. 2005). The $D_{4}$ band, which appears as a shoulder on the low wavenumber side of the $D$ band, and which seems to grow with the milling time, can eventually be attributed to hydrocarbon or aliphatic moieties connected on graphitic basic structural units (e.g. Bokobza et al. 2015; Sadezky et al. 2005). The relatively intense and broad bumpy second order bands $\left(2 D^{\prime}, D+G, 2 D\right)$ are indicative of the presence of numerous structural defects in the milled analogues, with the possible contribution at $2920 \mathrm{~cm}^{-1}$ of sp3 $\mathrm{CH}_{x}$ to the $D+G$ band (Ferrari \& Robertson 2001).

The infrared spectra of the many ball-milled precursor experiments, at long milling times, using different milling media and conditions are shown in the left panel of Fig. 6. High-resolution transmission electron microscope images for some of the samples are given in Fig. 7 to follow the structural evolution of the samples under milling. A comparison of absorption spectra with the infrared emission spectra of astronomical sources is shown in the right panels of Fig. 6. To perform such a comparison, the emission spectra of the astronomical sources already presented in Fig. 1 were divided by an emission grey-body spectrum, which was estimated by fitting a spline on the continuum outside the bands, at about $3300,2600,1800,1000$, and $550 \mathrm{~cm}^{-1}$. This is equivalent to assuming that the aromatic infrared emission band carriers emit at the same temperature as the underlying bands' continuum in this wavelength range. This step is necessary for a first-order comparison of the relative intensities to the one measured in absorption for the analogues in the laboratory. In particular, we note that when this emission correction is applied, the long wavelength polyaromatic $\mathrm{CH}$ out of plane modes (in the $700-900 \mathrm{~cm}^{-1}$ range) appears comparatively weaker than the 

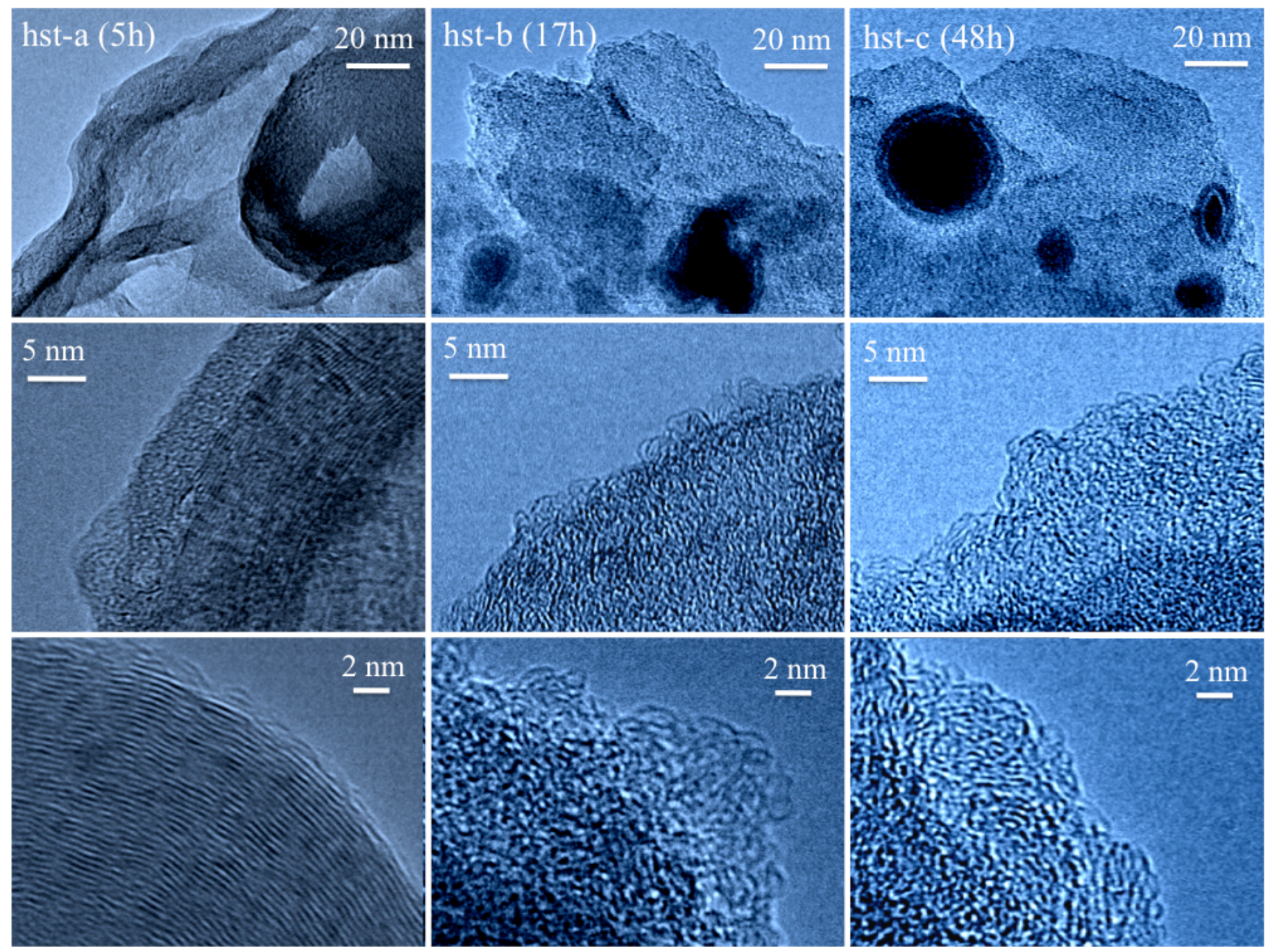

Fig. 4. High-resolution transmission electron microscope images at increasing magnification scales showing the structural evolution of ball-milled graphite under hydrogen atmosphere for the hardened steel experiment in a planetary ball mill after $5 \mathrm{~h}$ (left panels), $17 \mathrm{~h}$ (middle panels) and $48 \mathrm{~h}$ (right panels). The ordered graphitic plane evolves rapidly toward a turbostratic structure, and, at high milling times, a fully disordered carbon network with a high curvature. We note the progressive contamination of the hydrogenated carbon structure by iron nanoparticles issued from the shattering of the bowl during the milling process, giving rise to dispersed isolated passivated iron nanoparticles.

other vibrational modes and than what is observed in the raw astronomical emission spectra. The relative intensities are then closer to the one measured in the laboratory for the analogues. A zoom in on the out-of-plane aromatic $\mathrm{CH}$ modes is presented in Fig. 8, together with representative spectra of the analogues in different bowl-milling media. Several diagnostics are placed on these data. The integrated optical depth ratio of the $\mathrm{CH}_{x=2,3}$ modes in the stretching region from about 2800 to $2980 \mathrm{~cm}^{-1}$, hereafter called $2920 \mathrm{~cm}^{-1}$ to the corresponding deformation modes, $\mathcal{R}=2920 \mathrm{~cm}^{-1} /\left(1460 \mathrm{~cm}^{-1}+1375 \mathrm{~cm}^{-1}\right)$, varies only slightly in the analogues produced with $\mathcal{R}=5.9 \pm 1.4$. The integrated absorption of the aromatic $\mathrm{C}=\mathrm{C}$ band at $1600 \mathrm{~cm}^{-1}$, composite aliphatic $\mathrm{CH}_{x=2,3}$ band at about $1460 \mathrm{~cm}^{-1}$, and $\mathrm{CH}_{3}$ band at about $1375 \mathrm{~cm}^{-1}$ are evaluated, both in the analogues and the astronomical normalised spectra. These bands give access to the aliphatic $\mathrm{C}-\mathrm{H}$ to aromatic $\mathrm{C}=\mathrm{C}$ content of the samples and sources. The analysis focuses on the $1600-1000 \mathrm{~cm}^{-1}$ range. This range is chosen intentionally to build analytical ratio for several reasons. Firstly, as they are close in energy, their relative value in emission is expected to be least affected by temperature in the case of comparisons to astronomical spectra. Indeed, as can be seen from Fig. 1, the three-micron continuum slope can be opposite (e.g. IRAS 04296) to that of the continuum emission at a longer wavelength. It suggests that the emission arising at such wavelengths may come from an inner part, from a smaller distance to the astronomical source, and this spectral range cannot easily be compared to the mid infrared emitted from another region without precise knowledge of the source environment's geometry. The large band evolving between 1170 and $1350 \mathrm{~cm}^{-1}$ is also interesting, as it is an important diagnostic of the spectral evolution of the AIB's astronomical classes. Its potential assignments are discussed in the next section. The position of the maximum of this band in both observations and laboratory analogues are measured.

From these evaluations, the $1375 \mathrm{~cm}^{-1} \mathrm{CH}_{x=2,3}$ to $1460 \mathrm{~cm}^{-1}$ $\mathrm{CH}_{x=2,3}$ ratio is plotted in function of the $1600 \mathrm{~cm}^{-1} \mathrm{C}=\mathrm{C}$ to $1460 \mathrm{~cm}^{-1} \mathrm{CH}_{x=2,3}$ ratio in Fig. 9, for the class $C$ and $\mathcal{D}$ sources, a-C:H diffuse ISM sources for comparison, and the mechanochemically produced analogues. In the dust grain analogues, the $1600 \mathrm{~cm}^{-1} \mathrm{C}=\mathrm{C}$ to $1460 \mathrm{~cm}^{-1} \mathrm{CH}_{x=2,3}$ ratio is plotted in function of the measured elemental $\mathrm{H} / \mathrm{C}$ ratio in Fig. 10. The range for the considered observations is over-plotted for 


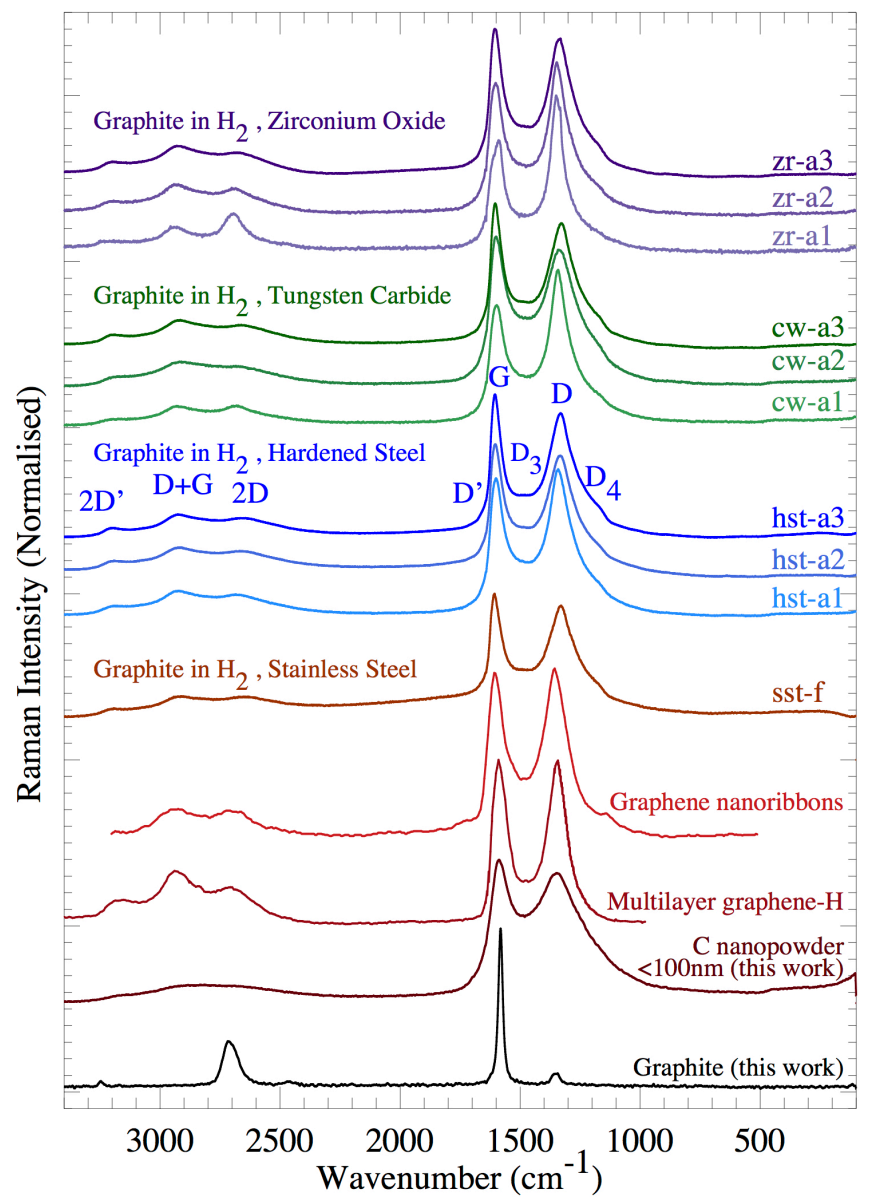

Fig. 5. Raman spectra of a sample set of planetary ball-milled graphite under a pressurised hydrogen atmosphere in function of milling time. The millings were performed in bowls made of stainless steel, hardened steel, tungsten carbide, and zirconium oxide. The Raman intensities were normalised. A graphite chunk and carbon nanopowder were measured for comparison. The spectra recorded for hydrogenated multilayer graphene (Ilyin et al. 2011) and graphene nanoribbons (Jovanović et al. 1996) are displayed.

comparison. In addition, the " $1300 " \mathrm{~cm}^{-1}$ ("7.7 $\mu \mathrm{m}$ ") massif peak maximum position is measured in the analogues and observations. Its position with regard to the elemental $\mathrm{H} / \mathrm{C}$ ratio is displayed in Fig. 11.

\section{Discussion}

During the milling process of carbonaceous precursors, the energies are high enough to shatter and break particles by highfrequency impact and intensive frictions, reducing their sizes down to hundreds of nanometres, and also profoundly affecting the samples at the atomic bonds level. In the presence of a hydrogen atmosphere pressurisation of the bowls, in addition to structural modifications, chemical reactions proceed, and hydrogenation of the produced particles' reactive sites further modifies the dust particles. This process is particularly attractive for forming astrophysical analogues, as it might circumvent one of the issues when producing laboratory analogues, namely the fact that in many occurrences, the analogue modifications are produced at a macroscopic scale. This high-impact/shattering milling process produces and hydrogenates defects very locally in the ball-milled carbonaceous dust particles, inducing multiscale defects.
The spectra of the produced analogues show marked similarities with astronomical observations. In particular, the analogues are able to provide a satisfactory spectral match to the $1300 \mathrm{~cm}^{-1}$ massif, together with large aliphatic deformation mode $(\sim 1460$ $\left.1375 \mathrm{~cm}^{-1}\right)$ to aromatic $\mathrm{C}=\mathrm{C}$ stretching mode $\left(\sim 1600 \mathrm{~cm}^{-1}\right)$ intensity ratios. Some of the bands are, however, still slightly broader than the astronomically observed ones. The $\mathrm{C}=\mathrm{C}$ mode shows an asymmetric profile warped toward longer wavelengths as observed in astronomical spectra.

The out-of-plane $\mathrm{CH}$ modes in the produced analogues, peaking at about $880 \mathrm{~cm}^{-1}$ and attributed to isolated solo $\mathrm{H}(1 \mathrm{H})$, at 832 and $808 \mathrm{~cm}^{-1}$ and attributed to duos $\mathrm{H}(2 \mathrm{H})$, at $755 \mathrm{~cm}^{-1}$ and attributed to trios or quartets $\mathrm{H}(3 \mathrm{H}, 4 \mathrm{H})$, are close to the astronomically observed peak positions and in agreement with their relative intensities. The prevalence of the aromatic solo $\mathrm{H}$ out of plane mode at about $880 \mathrm{~cm}^{-1}$ in class $C$ to $\mathcal{D}$ astronomical spectra is reproduced. This band possesses an intrinsically larger band strength than the other out-of-plane modes (e.g. Bauschlicher et al. 2009), by a factor up to about 4. If the integrated intensities in the duo modes $\left(832,808 \mathrm{~cm}^{-1}\right)$ are lower, the duos may contribute equally to the underlying structure. By contrast, the low intensities in the trio/quartet bands region of the spectrum supports the idea of hydrogen bonded to isolated or peripheral hydrogen $(1 \mathrm{H})$, or $(2 \mathrm{H})$ structures, as well as a rather low hydrogen coverage in the produced analogues.

There are ample discussions and research regarding the relation between the polyaromatic structure, intensity, and position of the $\mathrm{CH}$ the out-of-plane bands in the literature, (e.g. Bauschlicher et al. 2009; Candian et al. 2014; Russo et al. 2014; Tommasini et al. 2016; Sasaki et al. 2018; Ricca et al. 2019). For the $2 \mathrm{H}$ structure, the position can be typically associated with zigzag, armchair, or bay configurations.

The analogues' relative intensities fit the lower energy region of the spectra well. The $6.2 \mu \mathrm{m} / 6.85 \mu \mathrm{m}\left(1600 \mathrm{~cm}^{-1} / 1460 \mathrm{~cm}^{-1}\right)$ ratio that fit the observations is lower than what is expected when compared to the $\mathrm{CH}$ stretching-mode-region observations. In the $\mathrm{CH}$ stretching mode region, the aromatic $\left(\sim 3040 \mathrm{~cm}^{-1}\right)$ to aliphatic $\left(\sim 2760-2980 \mathrm{~cm}^{-1}\right) \mathrm{CH}$ bands intensities are higher, by a factor of a few units, in the sources observed (IRAS 042963429, IRAS 05341+0852, AFGL2688, IRAS 22272+5435, see upper-right panel of Fig. 6) than in the analogues. It suggests that the emission in this spectral range may, as mentioned above, arise in regions closer to the central source, with species possessing a slightly higher aromatic $\mathrm{CH}$ content.

The comparison between the observations' intensity ratios of the aromatic $\mathrm{C}=\mathrm{C}$ band at $1600 \mathrm{~cm}^{-1}$ to aliphatic bands at $1460 \mathrm{~cm}^{-1}$, versus the 1375 -to- $1460 \mathrm{~cm}^{-1}$ ratio immediately shows that these ratios are definitely different from the Galactic hydrogenated amorphous component observed in absorption in the diffuse interstellar medium (Fig. 9). On the contrary, these ratios agree fairly well with the analogues produced in this study. The lower 1375 -to- $1460 \mathrm{~cm}^{-1}$ ratio is an interesting criterion characterising a low(er) methyl-to-methylene ratio in the aliphatic $\mathrm{CH}$ inserted in the structure (although the exact relative intensities could be affected by a high stress in the material). $\mathrm{CH}_{2}$ may bridge faults in the aromatic structure.

The ratio of the aromatic $\mathrm{C}=\mathrm{C}$ band at $1600 \mathrm{~cm}^{-1}$ to the aliphatic $\mathrm{CH}_{x=2,3}$ band at $1460 \mathrm{~cm}^{-1}$ (Fig. 10), does not evolve linearly with the elemental hydrogen-to-carbon ratio (measured independently). In the milling process it implies that the first hydrogen atoms chemically attached form mainly aromatic $\mathrm{C}-\mathrm{H}$ bonds at early times. The aliphatic $\mathrm{C}$-Hs are formed in the next chemical attack steps, probably at the carbon sites destabilised by the first inserted hydrogens. 

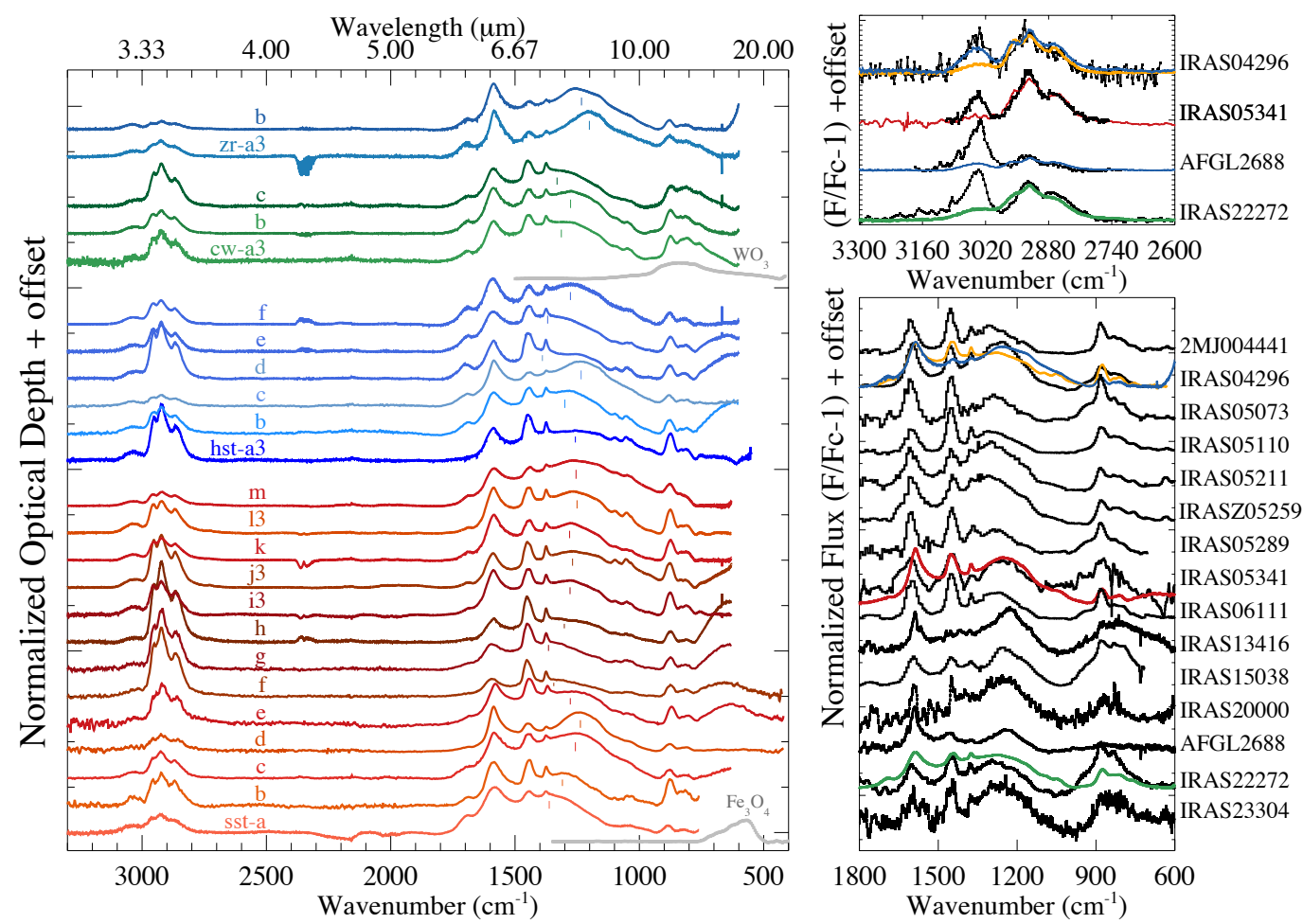

Fig. 6. Left panel: infrared spectra of the mechanochemically produced interstellar analogues, baseline subtracted. The colours correspond to different milling materials (stainless steel: red; hardened steel: blue; tungsten carbide: green; zirconium oxide: cyan). Infrared spectra of potential main contaminant for long-duration milling times are shown for clarity: iron oxide nanoparticles $\left(\mathrm{Fe}_{3} \mathrm{O}_{4}\right.$ spectrum from Can et al. 2010) for stainless steel and hardened steel and tungsten oxide $\left(\mathrm{WO}_{3}\right.$ spectrum from Dawson et al. 2011), expected to be marginal due to its high shear resistance, for tungsten carbide. The thin lines indicate the estimated position of the peak maximum for the broad massif. The nomenclature refers to the description in Table 2. Right panels: astronomical observations for comparison to the analogue spectra. Top: near-infrared literature spectra available for four out of all the analysed sources. Bottom: mid-infrared Spitzer and ISO spectra of class $C$ and $\mathcal{D}$ type sources analysed. For the spectra including both near and mir spectra, a combination of analogue spectra are overlaid for comparison.
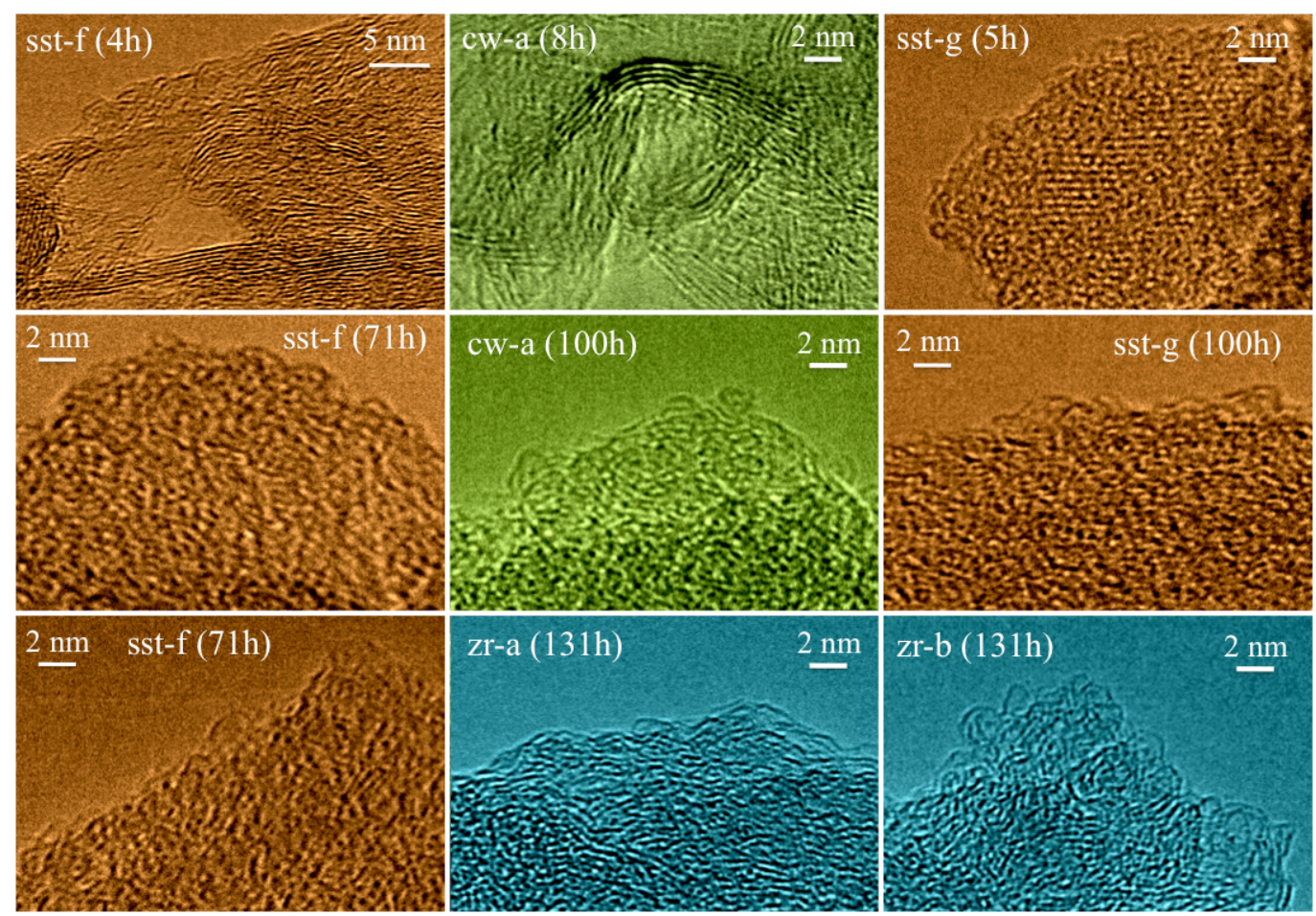

Fig. 7. High-resolution transmission electron microscope images for different ball-milled carbon precursors of this study and different mill materials. They are shown at large mechanochemical milling times performed in an hydrogen atmosphere. A few images are shown after a few hours of ball milling for comparison. The colour-coding for the images is brown for stainless steel, green for tungsten carbide, and light cyan for zirconium oxide experiments, respectively. 


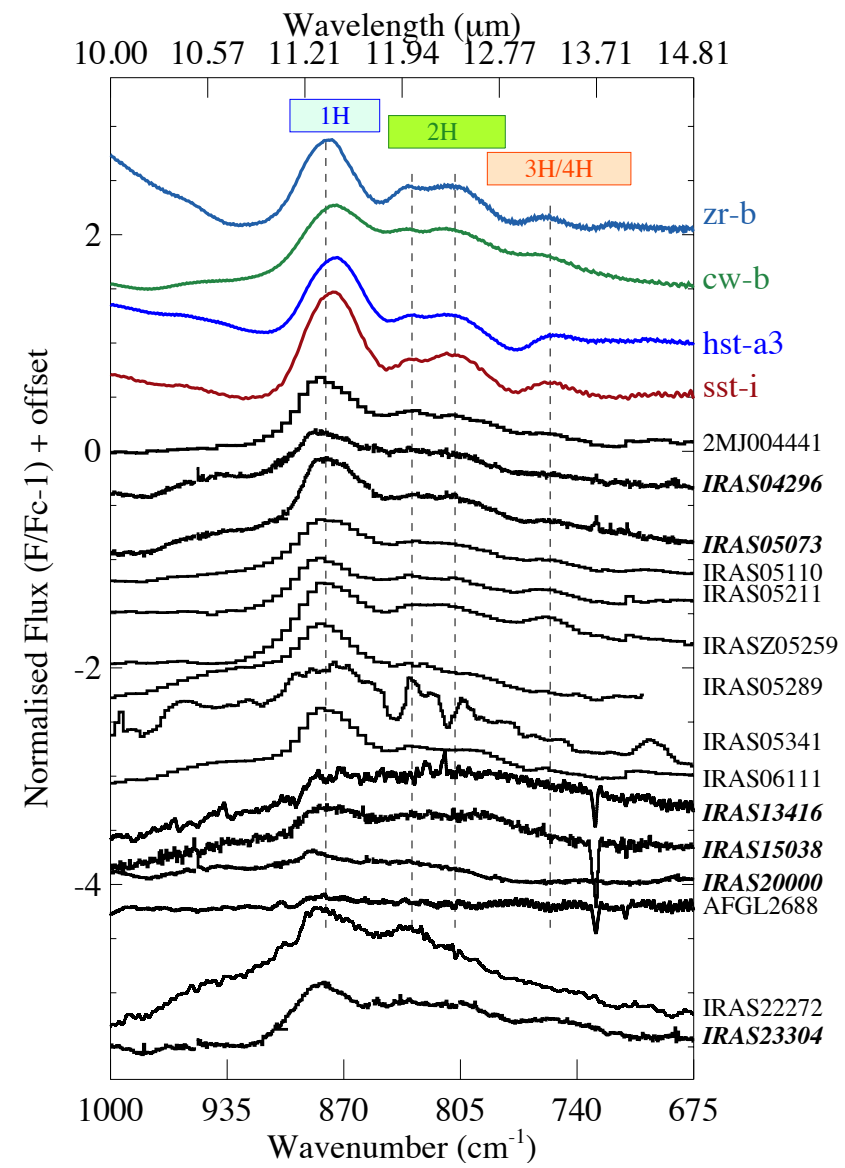

Fig. 8. Zoom on Spitzer and ISO spectra of class $C$ and $\mathcal{D}$ type sources analysed in the out-of-plane aromatic $\mathrm{CH}$ mode's spectral region. The sources labelled in italics correspond to available Spitzer highresolution spectra $(R \sim 600)$. Several analogues produced in different ball-milling environments are displayed above, for a direct comparison of the position of the observed bands and a range of measured relative intensities. The typical span of expected positions for the $\mathrm{CH}$ out of plane bands according to the underlying aromatic structure to which they are attached are given above $(1 \mathrm{H}$ : solo; $2 \mathrm{H}$ : duo-bay, armchair; $3 \mathrm{H}$ : trio; 4H: quartet).

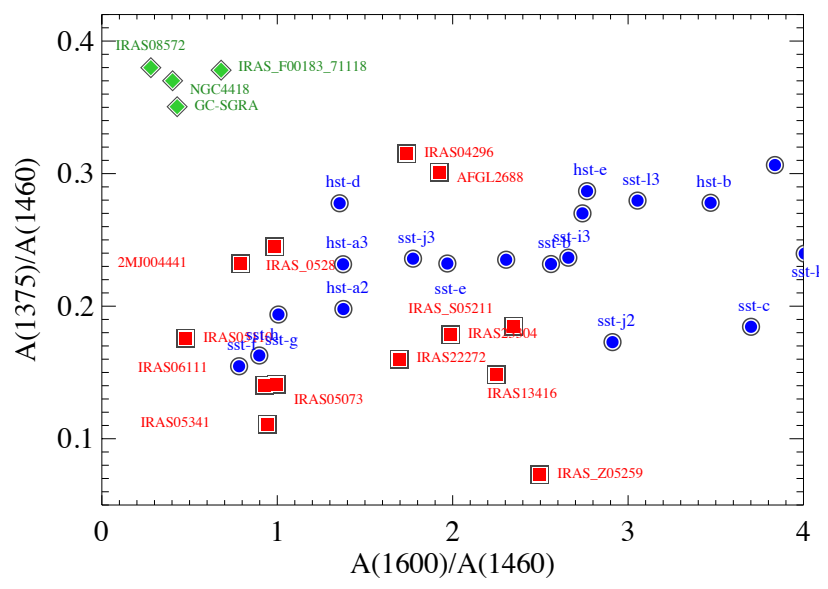

Fig. 9. Comparison between astronomical spectra integrated-intensity ratios of aromatic $\mathrm{C}=\mathrm{C}$ band at $1600 \mathrm{~cm}^{-1}$ to aliphatic $\mathrm{CH}_{x}$ band at $1460 \mathrm{~cm}^{-1}$ according to the aliphatic $\mathrm{CH}_{x} 1375 \mathrm{~cm}^{-1} / 1460 \mathrm{~cm}^{-1}$ ratio. The green diamonds correspond to the diffuse interstellar medium a-C:H absorptions. The red squares correspond to the class $C$ and $\mathcal{D}$ sources in this study. The blue dots are the same ratios measured on the infrared spectra of the produced analogues.

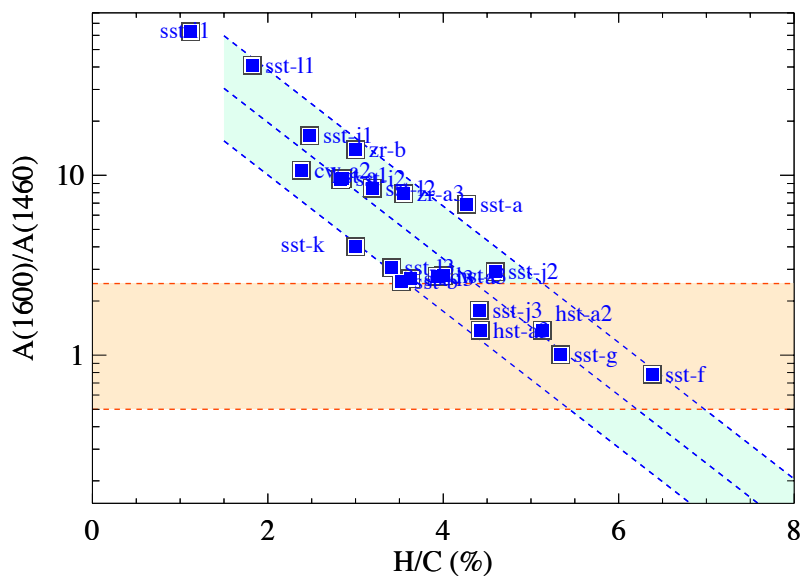

Fig. 10. Comparison between analogue spectra integrated-intensity ratios of aromatic $\mathrm{C}=\mathrm{C}$ band at $1600 \mathrm{~cm}^{-1}$, and aliphatic $\mathrm{CH}_{x}$ band at $1460 \mathrm{~cm}^{-1}$ as a function of the elemental hydrogen to carbon ratio measured independently. The blue coloured region encloses the confidence interval of the correlation. The orange coloured region corresponds to the observed range for astronomical observations.

The amount of material produced by this method made it possible to perform an elemental analysis to determine the $\mathrm{H} / \mathrm{C}$ ratio for a subset of the analogues. A comparison between the $1600 / 1460 \mathrm{~cm}^{-1}$ ratio in the analogues versus their $\mathrm{H} / \mathrm{C}$ content is presented in Fig. 10. The corresponding range measured on the astronomical spectra is shown in light orange. The agreement between the spectra and the observations is obtained for an hydrogen to carbon ratio of $5 \pm 2 \%$, setting a strong constraint.

The emission massif in the $1170-1300 \mathrm{~cm}^{-1}$ range (from about 8.6 up to $7.6 \mu \mathrm{m}$ ) is one of the spectral structures that is among the most specific for the AIB carriers, with a marked evolution from the class $\mathcal{A}$ to $\mathcal{D}$ (e.g. van Diedenhoven et al. 2004; Matsuura et al. 2014). In the produced analogues, the vibrational modes absorbing in this range can be associated with geometrical distortions of the C-C skeleton (e.g. Centrone et al. 2005; Lazzarini et al. 2016, and references therein), in addition to potential moderate $\mathrm{C}-\mathrm{O}-\mathrm{C}$ oxygen contamination mode contributions in the wing of the profile, as they peak mainly in the lower part of this wavenumber range. The position of the $1300 \mathrm{~cm}^{-1}$ massif thus puts a strong constraint on the internal structure or carbon based skeleton of the AIBs carriers. In the spectral evolution shown in Fig. 3 this massif evolves gradually from about $1200 \mathrm{~cm}^{-1}$ after a few hours to $1270-1350 \mathrm{~cm}^{-1}$ at large milling times. Its position is related to the onset of important curvature imposed by the production of nanoscale structures as sketched in Fig. 2. The HRTEM images of the ball-milled precursor at long milling time displayed in Fig. 7 show the ubiquitous nature of such warped and round curvatures with radii as small as 1 to $2 \mathrm{~nm}$. The position of the massif is reported in function of the $\mathrm{H} / \mathrm{C}$ ratio in Fig. 11. If a rather large dispersion still exists, this figure shows a positive correlation trend of the position with the $\mathrm{H} / \mathrm{C}$ content in the analogues. Thus, the chemical insertion of hydrogen influences (or betrays) the underlying carbon network structure.

\section{Conclusions}

Mechanochemistry represents a pertinent methodology to produce carbonaceous interstellar analogues, starting from pure carbon-based precursors immersed in a reactive hydrogen atmosphere. The impact and shear milling at high energy produces 


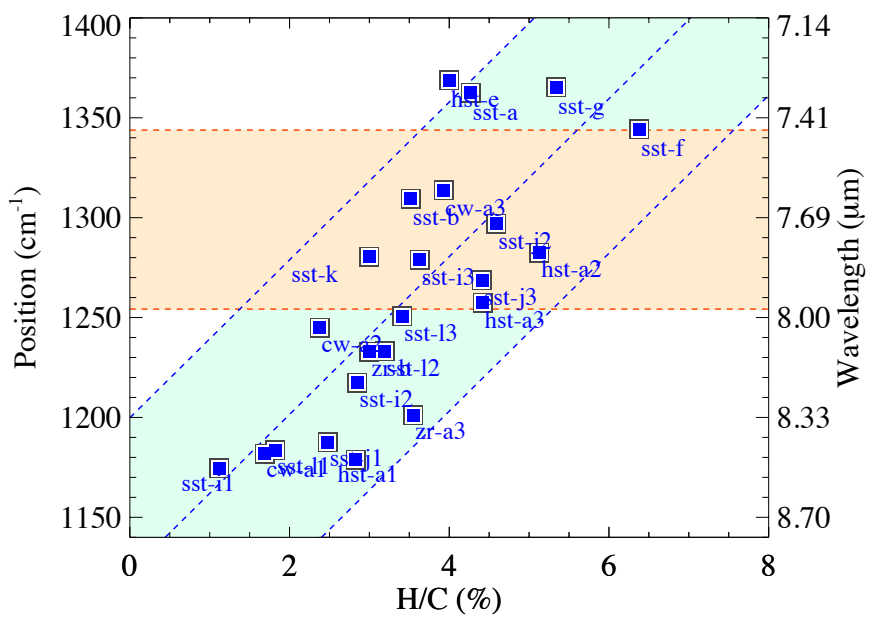

Fig. 11. Evolution of the " $1300 \mathrm{~cm}^{-1}$ " peak maximum position in analogue spectra displayed according to the elemental hydrogen to carbon ratio measured independently. The blue coloured region encloses the confidence interval of the correlation. The orange coloured region corresponds to the observed range for the astronomical observations analysed in this article.

local defects down to nanometre level and leads to a partial hydrogenation of resulting polyaromatic interlinked carbon structures. The main outcomes of this work are:

- These mechanochemical analogues successfully reproduce the overall spectra of polyaromatic infrared emission bands observed towards some astrophysical class $C$ and $\mathcal{D}$ type sources, including the poorly assessed and elusive broad emission band peaking in the 7.6-8.6 $\mu \mathrm{m}$ $\left(\sim 1170-1300 \mathrm{~cm}^{-1}\right)$ range, involving CC stretching and deformation modes.

- The position of the 7.6-8.6 $\mu \mathrm{m}$ band shows a trend related to the hydrogen to carbon content of the analogues produced, measured independently.

- A correlation is observed between the intensity ratio of the $\mathrm{C}=\mathrm{C}$ stretching at about $6.2-6.3 \mu \mathrm{m}\left(\sim 1600 \mathrm{~cm}^{-1}\right)$ and aliphatic C-H deformation at about $6.85 \mu \mathrm{m}\left(\sim 1460 \mathrm{~cm}^{-1}\right)$, and the hydrogen to carbon content.

- Transposed to astrophysical observations, the agreement between this ratio measured in analogues and observations in the mid-infrared range would imply the need for only about $3 \% \leq \mathrm{H} / \mathrm{C} \leq 7 \%$ in the polyaromatic-emitting structure; this represents a relatively low hydrogen content given the reducing $\mathrm{H}$-rich astrophysical media, to explain the observed spectra.

The mechanochemical approach to synthesise astrophysical carbonaceous dust particles, under an hydrogen atmosphere, is promising. It provides an adequate molecular structure for the reproduction of astronomical spectra, including the mid infrared bands. It enlightens us about the several percents hydrogen-tocarbon ratio in mixed aliphatic and aromatic carbonaceous dust required to explain the AIB observed emissions from class $C$ and $\mathcal{D}$ sources.

Acknowledgements. This work was supported by the INTEGRITY project, funded by the Domaine d'Intérêt Majeur ACAV, labelled by the Île-de-France region, the P2IO LabEx programme: "Evolution de la matière du milieu interstellaire aux exoplanètes avec le JWST", the ANR COMETOR project, grant ANR-18-CE31-0011 of the French Agence Nationale de la Recherche, and the Programme National "Physique et Chimie du Milieu Interstellaire" (PCMI) of CNRS/INSU with INC/INP co-funded by CEA and CNES. The Combined Atlas of Sources with Spitzer IRS Spectra (CASSIS) is a product of the IRS instrument team, supported by NASA and JPL. CASSIS is supported by the "Programme
National de Physique Stellaire" (PNPS) of CNRS/INSU co-funded by CEA and CNES and through the "Programme National Physique et Chimie du Milieu Interstellaire" (PCMI) of CNRS/INSU with INC/INP co-funded by CEA and CNES. Some scientific results reported in this article are based on observations made by the Infrared Space Observatory (ISO), an ESA science mission, and retrieved using the ISO database. We would like to thank J. Guigand, J.-P. Dugal, and $\mathrm{H}$. Bauduin for their support in the mechanical design modifications of the experiment used in this study. We would like to acknowledge the anonymous referee for the positive feedback on the article.

\section{References}

Acke, B., Bouwman, J., Juhász, A., et al. 2010, ApJ, 718, 558 Allamandola, L. J., Tielens, A. G. G. M., \& Barker, J. R. 1985, ApJ, 290, L25

Allen, D. A., \& Wickramasinghe, D. T. 1981, Nature, 294, 239

Bauschlicher, C. W., Peeters, E., \& Allamandola, L. J. 2009, ApJ, 697, 311

Biennier , L., Georges, R., Chandrasekaran, V., et al. 2009, Carbon, 47, 3295

Bokobza, L., Bruneel, J.-L., \& Couzi, M. 2015, Carbon, 1, 77

Boersma, C., Bouwman, J., Lahuis, F., et al. 2008, A\&A, 484, 241

Cami, J., Bernard-Salas, J., Peeters, E., et al. 2010, Science, 329, 1180

Can, M. M., Ozcan, S., Ceylan, A., \& Firat, T. 2010, Mater. Sci. Eng. B, 172, 72

Candian, A., Sarre, P. J., \& Tielens, A. G. G. M. 2014, ApJ, 791, L10

Carpentier, Y., Féraud, G., Dartois, E., et al. 2012, A\&A, 548, A40

Centrone, A., Brambilla, L., Renouard, T., et al. 2005, Carbon, 43, 1593

Dartois, E., \& Muñoz-Caro, G. M. 2007, A\&A, 476, 1235

Dartois, E., Muñoz Caro, G. M., Deboffle, D., et al. 2005, A\&A, 432, 895

Dartois, E., Geballe, T. R., Pino, T., et al. 2007, A\&A, 463, 635

Dawson, G., Zhou, W., \& Blackley, R. 2011, Phys. Chem. Chem. Phys., 13, 20923

Duley, W. W., \& Williams, D. A. 1983, MNRAS, 205, 67P

Ferrari, A. C., \& Robertson, J. 2001, Phys. Rev. B, 64, 075414

Francke, M., Hermann, H., Wenzel, R., Seifert, G., \& Wetzig, K. 2005, Carbon, 43, 1204

Furton, D. G., Laiho, J. W., \& Witt, A. N. 1999, ApJ, 526, 752

Gadallah, K. A. K., Mutschke, H., \& Jäger, C. 2013, A\&A, 554, A12

Geballe, T. R., Tielens, A. G. G. M., Kwok, S., et al. 1992, ApJ, 387, L89

Goto, M., Gaessler, W., Hayano, Y., et al. 2003, ApJ, 589, 419

Ilyin, A. M., Guseinov, N. R., Tsyganov, I. A., et al. 2011, Physica E LowDimensional Systems and Nanostructures, 43, 1262

Imanishi, M., Nakagawa, T., Shirahata, M., et al. 2010, ApJ, 721, 1233

Jäger, C., Krasnokutski, S., Staicu, A., et al. 2006, ApJS, 166, 557

Joblin, C., Tielens, A. G. G. M., Allamandola, L. J., et al. 1996, ApJ, 458, 610

Jovanović, S., Da Ross, T., Ostric, A., D Tošić, Prekodravac, D. J. , Marković, Z., \& Todorović Marković, B. 2014, Phys. Scr., T162, 14023

Keller, L. D., Sloan, G. C., Forrest, W. J., et al. 2008, ApJ, 684, 411

Kwok, S. 2016, A\&ARv, 24, 8

Lazzarini, A., Piovano, A., Pellegrini, R., et al. 2016, Catal. Sci. Technol., 6, 4910 Leger, A., \& Puget, J. L. 1984, A\&A, 500, 279

Mason, R. E., Wright, G., Pendleton, Y., et al. 2004, ApJ, 613, 770

Matsuura, M., Zijlstra, A. A., Bernard-Salas, J., et al. 2007, MNRAS, 382, 1889 Matsuura, M., Bernard-Salas, J., Lloyd Evans, T., et al. 2014, MNRAS, 439, 1472

Mennella, V., Brucato, J. R., Colangeli, L., et al. 1999, ApJ, 524, L71

Peeters, E., Hony, S., Van Kerckhoven, C., et al. 2002, A\&A, 390, 1089

Pino, T., Dartois, E., Cao, A.-T., et al. 2008, A\&A, 490, 665

Ricca, A., Roser, J. E., Peeters, E., et al. 2019, ApJ, 882, 56

Risaliti, G., Maiolino, R., Marconi, A., et al. 2006, MNRAS, 365, 303

Russo, C., Stanzione, F., Tregrossi, A., \& Ciajolo, A. 2014, Carbon, 74, 127

Sadezky, A., Muckenhuber, H., Grothe, H., Niessner, R., \& Pöschl, U. 2005, Carbon, 43, 1731

Sadjadi, S., Zhang, Y., \& Kwok, S. 2015, ApJ, 807, 95

Salver-Disma, F., Tarascona, J.-M., Clinard, C., \& Rouzaud, J.-N. 1999, Carbon, 37, 1941

Sasaki, T., Yamada, Y., \& Sato, S. 2018, Anal. Chem., 90, 18, 10724

Schnaiter, M., Henning, T., Mutschke, H., et al. 1999, ApJ, 519, 687

Sellgren, K., Werner, M. W., \& Ingalls, J. G. 2009, AAS Meeting Abstracts 214, 402.12

Sellgren, K., Werner, M. W., Ingalls, J. G., et al. 2010, ApJ, 722, L54

Sloan, G. C., Jura, M., Duley, W. W., et al. 2007, ApJ, 664, 1144

Szczerba, R., Siódmiak, N., \& Szyszka, C. 2005, Planetary Nebulae as Astronomical Tools (Matyland: AIP), 214

Tallant, D., Friedmann, T., Missert, N., Siegal, M., \& Sullivan, J. 1998, MRS Proc., 498

Tommasini, M., Lucotti, A., Alfè, M., et al. 2016, Spectrochim. Acta Pt. A Mol. Spectr., 152, 134

van Diedenhoven, B., Peeters, E., Van Kerckhoven, C., et al. 2004, ApJ, 611, 928

Yang, X. J., Glaser, R., Li, A., et al. 2016, MNRAS, 462, 1551 